\title{
Smoking history is negatively associated with allergen specific immunotherapy efficacy: a retrospective analysis
}

\author{
Jan Romantowski, Krzysztof Specjalski, Jakub Łata, Eliza Wasilewska, Marta Chełmińska, Ewa Jassem, \\ Marek Niedoszytko
}

Department of Allergology, Medical University of Gdansk, Gdansk, Poland

Adv Dermatol Allergol 2019; XXXVI (6): 673-676

DOI: https://doi.org/10.5114/ada.2018.80654

\begin{abstract}
Introduction: Allergen specific immunotherapy (AIT) is the only treatment modifying the course of the disease in patients allergic to airborne allergens. It has been proven to be effective in allergic populations, however individual patients vary in terms of response to the therapy.

Aim: To assess the factors that might affect the efficacy of AIT.

Material and methods: Patients treated with AIT for grass pollen or house dust mites were included. The efficacy of AIT was assessed with the use of Allergy Control Score (ACS), performed before and at least 1 year after AIT. The following variables were assessed as potential risk factors for a worse response to AIT: age, gender, type of allergy, type of allergen, type of vaccine, type of AIT and smoking history.

Results: The study group consisted of 145 subjects. AIT was effective in the entire group; the mean ACS results decreased from 21.14 to 14.41 points $(p<0.0001)$. No differences in efficacy in terms of assessed risk factors were found, except for smoking history (ACS change in the smoking group was smaller: from 21.8 to 18.1 points; $p=0.09$, $\mathrm{OR}=0.323 ; 95 \% \mathrm{Cl}: 0.11-0.88 ; p=0.02$ ).

Conclusions: Smoking history may affect AIT outcomes.
\end{abstract}

Key words: rhinitis, grass pollen, house dust mite, tobacco.

\section{Introduction}

Allergen specific immunotherapy (AIT) has been used for more than 100 years and is considered effective treatment This is the only disease modifying treatment for allergic diseases such as allergic rhinitis and asthma, with efficacy proven in numerous studies including randomized, double-blind placebo controlled clinical trials [1]. It should be emphasized that the treatment not only relieves typical hay fever symptoms, but also prevents asthma development, particularly in younger subjects [2]. Although generally successful, AIT might not be as efficient in particular patient subgroups such as smokers. However, data on the influence of smoking on AIT are highly limited.

\section{Aim}

The aim of the study was to assess factors that might affect the efficacy of AIT in patients treated for house dust mite or grass pollen allergies as those are two most prevalent airborne allergen sensitizations in Poland with particular focus on smoking.

\section{Material and methods}

The study group consisted of randomly chosen patients treated with AIT for at least 1 year since the start of AIT in the Outpatient Department of Allergology in Gdansk (Poland). Patients were allergic to grass pollen or house dust mites, which was confirmed by either positive skin prick tests or specific IgE. Allergic rhinitis was considered an indication for therapy diagnosed based on severe symptoms and lack of response to pharmacotherapy as described in ICON [3]. Atopic dermatitis and asthma were not considered a sole indication for treatment, however were not an excluding condition. Standard build up protocols were used.

For each patient the following data were recorded: age, gender, type of allergy (rhinitis, asthma, or both), type of allergen (pollen or house dust mites), type of vac-

Address for correspondence: Jan Romantowski MD, PhD, Department of Allergology, Medical University of Gdansk, 7 Dębinki St, 80-211 Gdansk, Poland, phone: +48 5834926 25, fax: +48 5834916 25, e-mail: jromant@gumed.edu.pl Received: 5.03.2018, accepted: 11.07.2018. 
cine (allergen extract or allergoids), type of AIT (SCIT or SLIT) and smoking history. In addition, age at allergy diagnosis, past and current treatment and co-morbidities, such as hypertension, gastroesophageal reflux or hypothyroidism were also noted.

To assess the efficacy of AIT, patients received two Allergy Control Score (ACS): concerning symptoms and medication before AIT and at least 1 year after the initiation of AIT. Medication doses were calculated into points using the Reference Scoring List provided by AllergoPharma based on a non-commercial agreement [4].

The study was approved by the appropriate Bioethics Commission in Medical University of Gdansk. All patients consented to study participation.

\section{Statistical analysis}

The results were analysed using Wilcoxon's, U Mann Whitney's tests, multiple logistic regression (predictors: age, sex, asthma, atopic dermatitis, GERD, hypertension, hypothyroidism), and $\chi^{2}$ implemented in Statistica 12 software (Statsoft, U.S.A.). Normal distribution was not observed in the general population and subgroups, which was analysed using the Shapiro-Wilk test.

\section{Results}

In total 145 patients were included in the study (67 women and 78 men). The average age was 32.6 years (range: 18-52 years). The average duration of entire treatment at the moment of assessment was 1,076 days (range: 366-1969). The average age of diagnosis was 18.1 years and the mean interval between the first al- lergy symptoms and the start of AIT was 11 years. The total number of patients reporting co-morbidities was low (27) with gastroesophageal reflux as the most prevalent $(n=9)$, thus statistical analysis on those subgroups was not performed. The majority of patients $(n=146)$ received SCIT, while 2 patients were treated with SLIT. Twenty eight patients reported smoking history (at least 1 year of smoking), out of them 14 were still active smokers. Allergic rhinitis was diagnosed in all patients, while 61 (41\%) suffered also from asthma.

The mean ACS values for the studied group before and during immunotherapy were 21.44 and 14.41, respectively $(p<0.0001)$. No differences in the efficacy of AIT in terms of assessed risk factors were found, except for smoking history (ACS change from 21.8 to 18.1 points; $p=0.09$ ) (Figure 1). Multiple regression analysis proved the lower efficacy of AIT in smokers (OR $=0.31 ; 95 \% \mathrm{Cl}$ : $0.12-0.83 ; p=0.02$ ). In the smoking group, $42 \%$ of patients did not report any improvement in ACS compared to $20 \%$ of the non-smoking studied population ( $p=0.02$ ).

\section{Discussion}

The results support the efficacy of AIT, but at the same time showed that smoking history may negatively affect the clinical result.

Although no selection according to sex was applied, men prevailed in the study group. This gender difference has already been observed before. A study conducted in the United States on 102,390 American paediatric patients showed that boys were $25 \%$ more likely than girls to receive AIT $(p<0.0001)$ [5].

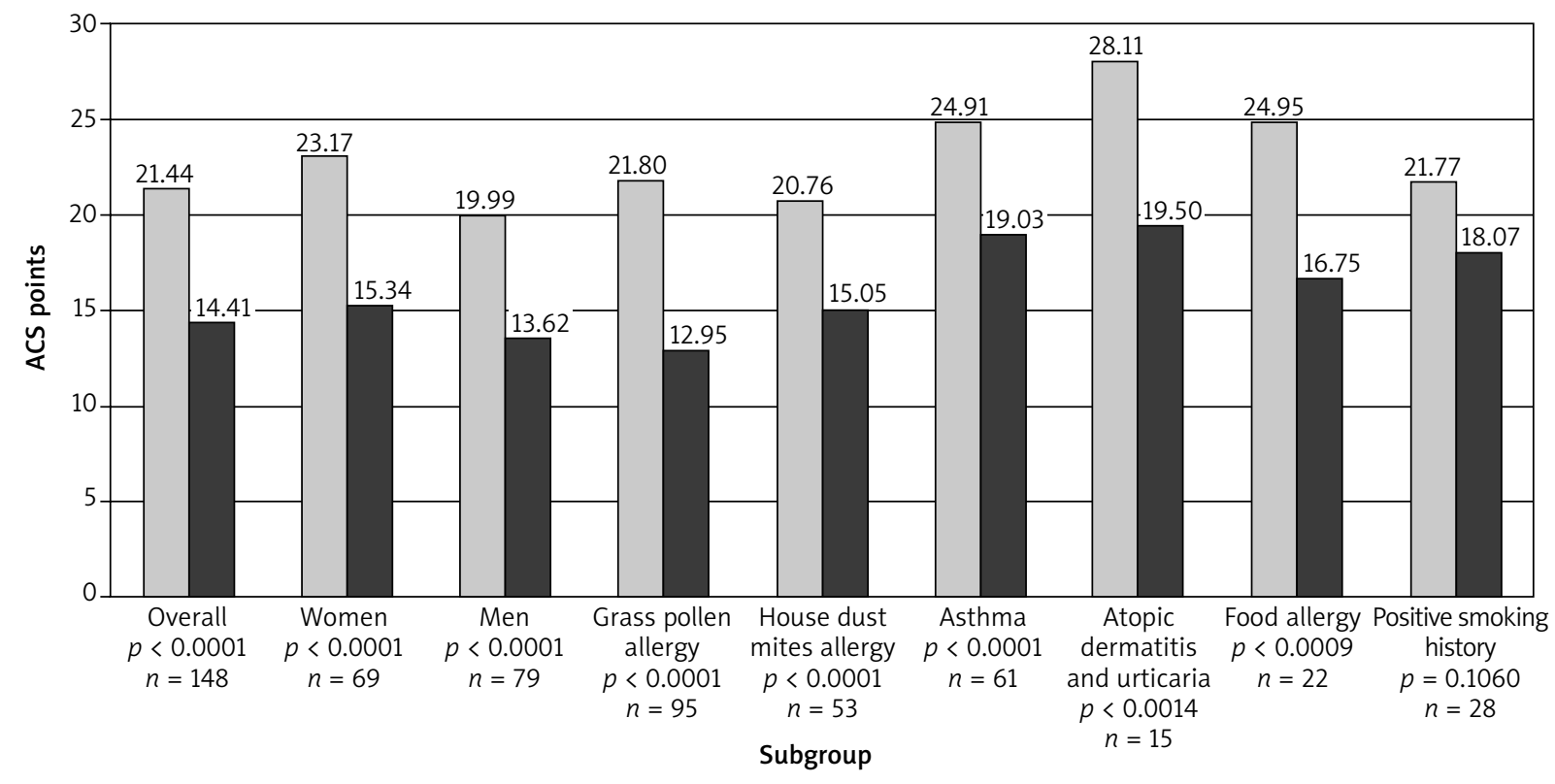

Figure 1. Average ACS before and after the start of SIT (ACS1 and ACS2, respectively). Subgroup analysis 
The most interesting finding was the lack of a significant effect of AIT in patients with a smoking history. This observation is in line with some previous studies. In a randomized study on 68 children receiving AIT, it was found that subjects exposed to passive smoking presented higher nasal eosinophilia, a poorer symptom-medication score followed by poor asthma control [6]. Analysis of data from the German National Health Interview and Examination Survey in 1998 including 298 subjects that have undergone AIT revealed that conduction and completion was more frequent in men that have never smoked. This may have been due to a better clinical response, however the study did not analyse that issue [7].

An obvious explanation for this phenomenon would be the influence of tobacco exposure on asthma control. It is known that smoking enhances asthma symptoms, worsens lung function and limits inhaled corticosteroids efficacy [8]. However, in this study only half of patients with smoking history were asthmatics. Data regarding the influence of smoking on IgE-mediated allergies and AIT are limited and some studies do not confirm the above findings. In a study of 163 patients on SLIT due to allergic rhinitis, no significant difference was observed in the quality of life of smokers and nonsmokers. A study on 99 children receiving SCIT also did not reveal an impact of passive smoking $[9,10]$. The influence of smoking on allergic rhinitis is still unclear. In epidemiological studies, smoking is commonly considered a significant risk factor for allergic rhinitis [11]. However, a cohort questionnaire study conducted in Sweden on 27,879 subjects revealed that smoking was related to a higher prevalence of chronic non-allergic rhinitis and lower prevalence of allergic rhinitis. This observation was confirmed in male participants only [12]. Furthermore, a study by Bousquet et al. on 1,444 subjects including $20.8 \%$ of smokers and $10.9 \%$ of exsmokers showed that smoking did not affect allergic rhinitis. There appeared to be no significant differences in subgroups according to severity of nasal symptoms and results on the Rhinoconjunctivitis Quality of Life Questionnaire evaluation [13]. On the other hand, a prospective study on 81 children with allergic rhinitis showed a tendency toward increased nasal obstruction on the Visual Analog Scale in subjects exposed to passive smoking. In this case, the smoking exposure was determined by measuring the urinary cotinine/creatinine ratio [14].

Smoking also has an interesting, though disputable impact on allergen sensitization. Several studies revealed that smokers were less likely to develop positive skin prick tests and/or specific IgE during follow-ups for several years [15]. The authors usually regard these observations to be a result of the immunosuppressive mechanism of smoking. These findings are in contrast to epidemiological studies that highlighted smoking as a risk factor for allergy and asthma [16]. Finally, a study on 2,714 children and adolescents showed that active and passive smoking increased the risk of rhinitis but decreased the risk of allergen sensitization [17]. Still, the impact of smoking on allergy development needs to be further investigated.

An additional explanation to the main finding of our study may include the psychological issues observed in smokers. A study conducted in Central Europe on 359 patients treated for hypertension showed that smokers had significantly lower compliance [18]. In addition, in 234 patients with periodontitis, smokers' lack of compliance was assessed at $42 \%$ compared to $31 \%$ in nonsmokers [19]. Finally, a study on 47 patients using a continuous positive airway pressure device as a treatment for obstructive sleep apnoea syndrome (OSAS) showed that active smokers had significantly lower device usage compliance [20].

Finally, several weak points of the study should be mentioned, including the subjective method of assessing the efficacy of AIT. Moreover, the study is a one-centred, small analysis; therefore all findings warrant further confirmation.

\section{Conclusions}

Smoking is negatively associated with AIT.

\section{References}

1. Lockey RF. Historical perspectives of allergy and immunology and allergen immunotherapy. Ann Allergy Asthma Immunol 2014; 113: 587-92.

2. Schmitt J, Schwarz K, Stadler E, Wustenberg EG. Allergy immunotherapy for allergic rhinitis effectively prevents asthma: results from a large retrospective cohort study. J Allergy Clin Immunol 2015; 136: 1511-6.

3. Jutel M, Agache I, Bonini S, et al. International consensus on allergy immunotherapy. J Allergy Clin Immunol 2015; 136 : 556-68.

4. Hafner D, Reich K, Matricardi PM, et al. Prospective validation of "Allergy-Control-SCORE(TM)": a novel symptommedication score for clinical trials. Allergy 2011; 66: 629-36.

5. Hankin CS, Cox L, Lang D, et al. Allergy immunotherapy among Medicaid-enrolled children with allergic rhinitis: patterns of care, resource use, and costs. J Allergy Clin Immunol 2008; 121: 227-32.

6. Marogna M, Massolo A, Colombo F, et al. Children passive smoking jeopardises the efficacy of standard anti-allergic pharmacological therapy, while sublingual immunotherapy withstands. Allergol Immunopathol 2011; 39: 60-7.

7. Hommers L, Ellert U, Scheidt-Nave C, Langen U. Factors contributing to conductance and outcome of specific immunotherapy: data from the German National Health Interview and Examination Survey 1998. Eur J Public Health 2007; 17: 278 LP-84.

8. Thomson NC. The role of environmental tobacco smoke in the origins and progression of asthma. Curr Allergy Asthma Rep 2007; 7: 303-9.

9. Katotomichelakis M, Tripsianis G, Daniilidi A, et al. Smoking effects on quality of life of allergic rhinitis patients after sublingual immunotherapy. Rhinology 2015; 53: 325-31. 
10. Peng W, Liu E. Factors influencing the response to specific immunotherapy for asthma in children aged 5-16 years. Pediatr Int 2013; 55: 680-4.

11. Alexandropoulos T, Haidich AB, Pilalas D, et al. Characteristics of patients with allergic rhinitis in an outpatient clinic: a retrospective study. Allergol Immunopathol 2013; 41: 194200.

12. Eriksson J, Ekerljung L, Sundblad BM, et al. Cigarette smoking is associated with high prevalence of chronic rhinitis and low prevalence of allergic rhinitis in men. Allergy Eur J Allergy Clin Immunol 2013; 68: 347-54.

13. Bousquet PJ, Cropet C, Klossek JM, et al. Effect of smoking on symptoms of allergic rhinitis. Ann Allergy, Asthma Immunol 2009; 103: 195-200.

14. De S, Fenton JE, Jones AS, Clarke RW. Passive smoking, allergic rhinitis and nasal obstruction in children. J Laryngol Otol 2005; 119: 955-7.

15. Linneberg A, Nielsen NH, Madsen F, et al. Smoking and the development of allergic sensitization to aeroallergens in adults: a prospective population-based study. The Copenhagen Allergy Study. Allergy 2001; 56: 328-32.

16. Zetterström O, Osterman K, Machado L, Johansson SG. Another smoking hazard: raised serum IgE concentration and increased risk of occupational allergy. Br Med J 1981; 283: 1215-7.

17. Shargorodsky J, Garcia-Esquinas E, Navas-Acien A, Lin SY. Allergic sensitization, rhinitis, and tobacco smoke exposure in U.S. children and adolescents. Int Forum Allergy Rhinol 2015; 5: 471-6.

18. Fodor GJ, Kotrec M, Bacskai K, et al. Is interview a reliable method to verify the compliance with antihypertensive therapy? An international central-European study. J Hypertens 2005; 23: 1261-6.

19. Modin C, Abadji D, Adler L, Jansson L. Treatment compliance in patients with aggressive periodontitis - a retrospective case-control study. Acta Odontol Scand 2017; 75: 94-9.

20. Boyaci H, Gacar K, Bariş SA, et al. Positive airway pressure device compliance of the patients with obstructive sleep apnea syndrome. Adv Clin Exp Med 2013; 22: 809-15. 